\title{
Elemente und Funktionen des Systemmodells
}

\section{Daniel Liedtke ${ }^{a}$, Ole Wiesinger ${ }^{b}$, Christian Westerhoff ${ }^{c}$, Urs Stoffeld ${ }^{\text {, }}$ Reto Stocker ${ }^{e}$, Stephan Pahls ${ }^{f}$}

${ }^{a}$ Dr., COO Privatklinikgruppe Hirslanden; ${ }^{b}$ Dr. med., CEO Privatklinikgruppe Hirslanden; ${ }^{c}$ Dr. med., CCO Privatklinikgruppe Hirslanden; ${ }^{d}$ Dr. med., Chirurgische Praxis Enge, Zürich; ${ }^{e}$ Prof. Dr. med., Institut für Anästhesiologie und Intensivmedizin Klinik Hirslanden, Zürich; ${ }^{\dagger}$ Dr. med., Pahls Consulting, Zürich

Das Modell propagiert klarere Gewaltentrennung und stärkere Arbeitsteilung zwischen Professionen und Betriebseinheiten. Wichtige Elemente sind ein zentrales Medizinisches Qualitäts- und Performance-Management, sowie die Unterscheidung zwischen Medizinischen Service-Einheiten als Grundversorger im Spital und Medizinischen Spezialisten-Einheiten, die in ihrer Sub- oder Super-Spezialisierung auf die differenzierteren Bedürfnisse von Patienten und Zuweisern einzugehen haben.

In der letzten Ausgabe der Schweizerischen Ärztezeitung haben wir ein Systemmodell für die Organisation der Ärzteschaft in Akutspitälern vorgestellt [1]. Die wichtigsten Attribute der zentralen Elemente des Modells gehen aus Abbildung 1 hervor. In diesem Artikel möchten wir ausgewählte Elemente des Modells näher beleuchten.

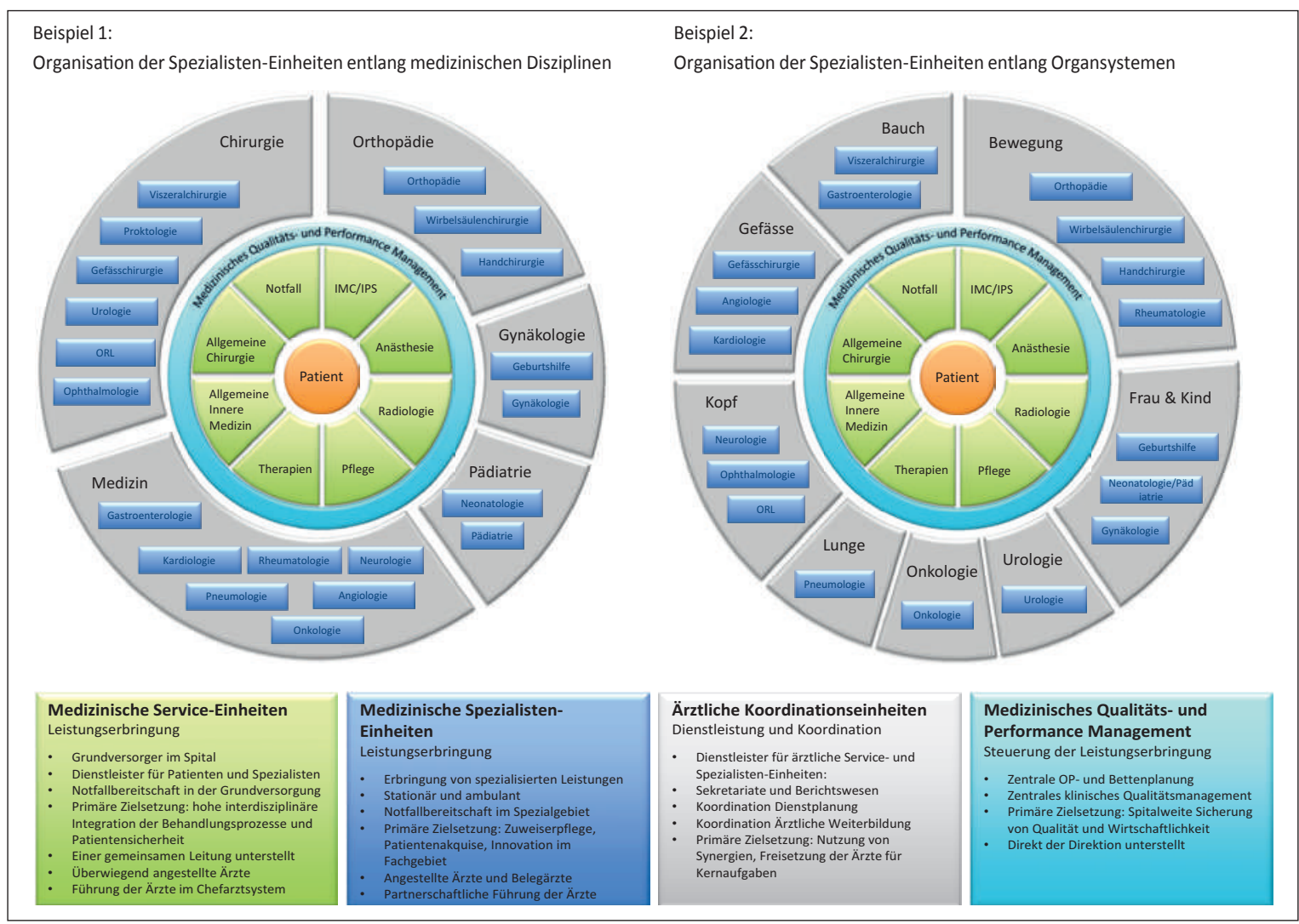

Abbildung 1: Möglichkeiten der Ausgestaltung des Systemmodells in Akutspitälern (Auswahl der Spezialisten-Einheiten nur beispielhaft).

\section{Ärztliche Kernaufgaben}

Ein Hauptanliegen des Systemmodells ist, den Spitalärzten mehr Raum für ihre Kernaufgaben zu geben. Dazu zählen wir die klinische Arbeit am Patienten, die ärztliche Fort- und Weiterbildung und die klinische Forschung und Innovation. Die Hauptaufgabe der Leitungen aller ärztlichen Service- und Spezialisten-

Beispiel 2:
Organisation der Spezialisten-Einheiten entlang Organsystemen

Ärztliche Koordinationseinheite enstleistung und Koordination

Dienstleister für ärztliche Service- und Sekretariate und Berichtswes Koordination Dienstplanung Koordination Arztliche Weiterbildun Synergien, Freisetzung der Ärzte fü Kernaufgaben 
Einheiten ist folglich die Sicherung hoher Standards in diesen Kernaufgaben in ihrem Bereich. Eine konsequente Fokussierung auf die Kernaufgaben wirkt sich unmittelbar positiv auf den Outcome und die Zufriedenheit von Patienten, Mitarbeitenden und Zuweisern aus, bedeutet aber auch, dass nicht mehr jeder Chefarzt in der Spitalleitung vertreten sein muss.

\section{Die Medizinischen Service-Einheiten}

In den Service-Einheiten beinhaltet die ärztliche Führungsverantwortung zusätzlich zu den Kernaufgaben vor allem die Themen Servicequalität, Prozessintegration und Patientensicherheit.

Die Service-Einheiten sind als Grundversorger im Spital medizinische Dienstleister für die Patienten und die Ärzte der Spezialisten-Einheiten. Sie betreiben die zentrale medizinische Infrastruktur des Spitals (Notfall, OP, Intensivstation usw.) und sind, in enger $\mathrm{Ab}$ stimmung mit den Spezialisten-Einheiten, massgeblich für den Patientenpfad von Eintritt bis Austritt und damit auch für die Patientensicherheit verantwortlich. Um eine hohe Verbindlichkeit und eine gemeinsame Servicekultur zu erreichen, werden alle Ärzte der Medizinischen Service-Einheiten im Chefarztsystem geführt und sind vom Spital angestellt. Diese Chefärzte, wie auch die Leitungspersonen der nicht-ärztlichen medizinischen Service-Einheiten, sind direkt einer gemeinsamen Leitung unterstellt, die direkt der Spitaldirektion zugeordnet, bzw. Mitglied der Spitalleitung ist.

Mögliche Interessenkonflikte zwischen den Leitern der Service-Einheiten können durch die unparteiische, zentrale Leitungsfunktion mit Bezug auf die gemeinsamen Zielgrössen Patientensicherheit, Patientenzufriedenheit und Prozesseffizienz gelöst werden. Diese Fokussierung auf gemeinsame Ziele im Sinne einer Corporate Culture bietet einen anerkannten Beurteilungsmassstab für die Lösung von Konflikten innerhalb der Service-Einheiten und vis-à-vis den Spezialisten-Einheiten.
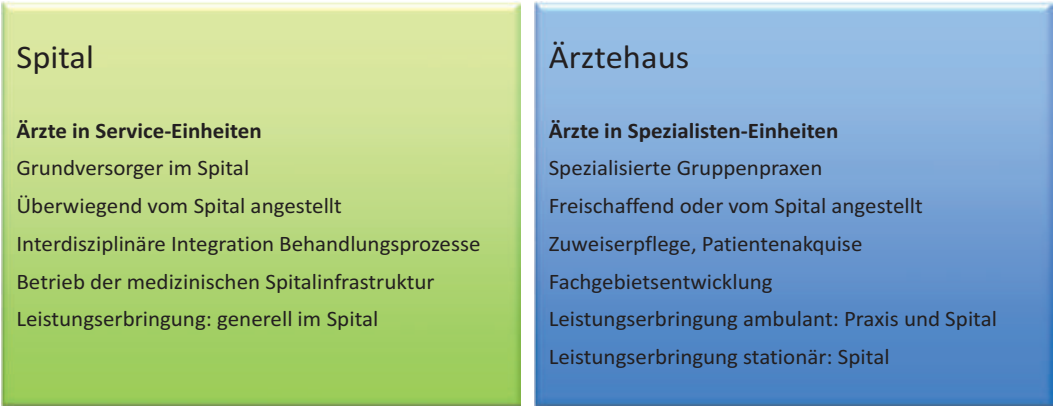

Abbildung 2: Beispiel der räumlichen Anordnung von ärztlichen Service- und Spezialisten-Einheiten in Spital und Ärztehaus.
Zu erwähnen ist auch die Bedeutung der ServiceEinheiten für die Rekrutierung ärztlicher Spezialisten. Die hohe fachliche Kompetenz, Servicequalität und Prozesseffizienz der integrierten Grundversorgung im Spital kann im zunehmenden Wettbewerb den entscheidenden Ausschlag für die erfolgreiche Bindung ärztlicher «Super-Spezialisten» an das Spital geben.

\section{Die Medizinischen Spezialisten-Einheiten}

In den Spezialisten-Einheiten beinhaltet die ärztliche Führungsverantwortung zusätzlich zu den Kernaufgaben vor allem die Zuweiserpflege und Patientenakquise, wozu die Schaffung einer patientenzentrierten Kultur innerhalb der Spezialisten-Einheit entscheidende Voraussetzung ist.

Viele Spezialisten erbringen einen grossen Teil ihrer Leistungen ambulant (Pneumologie, Onkologie usw.), leisten einen wichtigen Beitrag zur Reputation des Spitals und weisen Patienten für stationäre Behandlungen zu. Diese Ärzte sollen sich auf ihre Kernaufgaben konzentrieren und mit genügend Autonomie ausgestattet ihr Fachgebiet weiterentwickeln können. Die Ärzte der Spezialisten-Einheiten können vom Spital angestellt oder Belegärzte sein und sollten sich partnerschaftlich-kollegial organisieren können (z.B. Chairman-System, Partner-System, Chefarzt-System).

Die Unterbringung der Spezialisten-Einheiten in einem Ärztehaus auf oder in unmittelbarer Nähe des Spitalgeländes stellt eine interessante räumliche Lösung mit Vorteilen für Spital und Ärzte dar. Die ambulante Tätigkeit im Ärztehaus kann nahtlos mit der Behandlung von stationären Patienten im Spital kombiniert werden. Die räumliche Nähe erleichtert den interdisziplinären Austausch unter den Spezialisten, vereinfacht Patienten-Überweisungen und ist Bedingung für eine zeitnahe Intervention bei Notfällen und Komplikationen in komplexen Behandlungsfällen. Das Spital profitiert von einer breiten Verfügbarkeit von Subspezialitäten, ohne alle Spezialisten selbst anstellen zu müssen, und hat mehr Möglichkeiten, «Super»-Spezialisten mit hohem Renommee je nach persönlicher Präferenz flexible Beschäftigungslösungen anzubieten (Anstellung oder Belegarztstatus, Vollzeit oder Teilzeit usw.). Das Ärztehaus bündelt die Ressourcen der Spezialisten und erleichtert Teilzeitarbeit, was wiederum dem Fachärztemangel entgegenwirkt.

\section{Die Ärztlichen Koordinationseinheiten}

Die Koordinationseinheiten sind schlanke Dienstleistungseinheiten, die Aufgaben wie Dienstplanung, Be- 
richtswesen, ärztliche Fort- und Weiterbildung u.a. für eine Gruppe von fachlich verwandten Service- und Spezialisten-Einheiten koordinieren, dadurch Synergien nutzen, im nicht-klinischen Bereich Effizienzgewinne generieren und mehr Raum für die ärztlichen Kernaufgaben schaffen.

Die Führung der Koordinationseinheiten sollte grundsätzlich kollegial-koordinativ ausgerichtet sein, ist darüber hinaus aber vielseitig realisierbar. In kleineren Spitälern kann z.B. der Chefarzt einer Service-Einheit diese Rolle übernehmen, muss jedoch darauf achten, dass er die Aufgabe nicht als Rolle eines klassischen Klinik-Chefs interpretiert, sondern als Dienstleister für die zugeordneten Service- und Spezialisten-Einheiten. In grösseren Spitälern kann die Funktion z.B. von einer Fachperson mit direkter Unterstellung unter die Klinikdirektion erfüllt werden. Ein rotierendes Chairman-System stellt eine weitere Variante dar. Entscheidend für die Akzeptanz der Koordinationseinheiten ist auch hier die enge kollegiale Abstimmung mit den Spezialisten-Einheiten. Auf Stufe der Ärztlichen Koordinationseinheiten nimmt die ärztliche Fort- und Weiterbildung eine besonders wichtige Stellung ein. Eine vertiefte Diskussion dieser Aufgabe übersteigt jedoch den Rahmen der vorliegenden Publikation und soll bei geeigneter Gelegenheit separat erfolgen.

\section{Das Medizinische Qualitäts- und Performance-Management}

Das Medizinische Qualitäts- und Performance-Management nimmt eine Schlüsselstellung im Systemmodell ein und sollte direkt der Spitaldirektion unterstellt sein, um in ihrem Kompetenzbereich wirksame Weisungsbefugnis gegenüber Service- und Spezialisten-Einheiten zu gewährleisten. Bevorzugt wird die Funktion durch eine medizinische Fachperson mit betriebswirtschaftlicher Zusatzausbildung geleitet. Die Akzeptanz und Glaubwürdigkeit des Qualitäts- und PerformanceManagements steht und fällt mit der Besetzung durch Personen, die ihre Aufgaben integrativ und transparent in Abstimmung mit den Service- und Spezialisten-Einheiten ausführen.

\section{Zentrales Performance-Management}

Eine wichtige Prämisse des Systemmodells ist der Abschied vom Konzept der «bettenführenden Klinik» und der Hoheit der Operateure über die OP-Planung. Im Zeitalter der Fallpauschalen ist eine hohe OP- und Bettenauslastung für den wirtschaftlichen Erfolg eines Spitals entscheidend und kann nur durch eine zentrale und flexible Planung erreicht werden. Dies ist kaum zu erreichen, wenn Betten oder OP-Säle fix bestimmten
Kliniken zugewiesen sind und Chefärzte die Belegung steuern.

Im Systemmodell werden die Kapazitäten der ServiceEinheiten den im Spital tätigen Spezialisten und ihren Patienten nachfrageorientiert zur Verfügung gestellt. Das betrifft OP, Interventionssäle, Intensivstation und Bettenstationen gleichermassen. Die zentrale OP- und Bettendisposition stellt, in ständiger Absprache mit allen Einheiten, sicher, dass die Patienten in der geeigneten Infrastruktur mit der entsprechenden fachlichen Kompetenz betreut werden und gleichzeitig eine hohe Belegung erreicht wird.

\section{Dieses Konzept "Fallführung statt Betten-} führung» bedeutet einen fundamentalen Kulturwandel.

Indem auf diese Weise die Zuständigkeit der Fachärzte nicht mehr durch die Ressource «Bett» oder «OP-Saal», sondern durch die Fokussierung auf den Behandlungsfall definiert wird, können in einem bestimmten Operationssaal und auf einer bestimmten Bettenstation die Patienten mehrerer Spezialisten-Einheiten ohne Kompetenzkonflikte auf ärztlicher Seite betreut werden, was u.a. die Integration von Belegärzten erleichtert. Dieses Konzept «Fallführung statt Bettenführung» bedeutet einen fundamentalen Kulturwandel, der für nicht wenige Chefärzte in Spitälern mit der heute vorherrschenden Klinik-Struktur die Aufgabe von historisch gewachsenen Privilegien erfordert.

\section{Zentrales klinisches Qualitätsmanagement}

Praktisch alle Schweizer Spitäler haben das Qualitätsmanagement auf Stufe Unternehmen institutionalisiert und beschäftigen qualifizierte Qualitätsmanager/-innen, die den Klinikern viel Arbeit z.B. bei der Dokumentation ihrer Prozesse, bei der Implementierung von Qualitätssystemen (z.B. CIRS), bei der Spitalhygiene und bei Qualitätsmessungen abnehmen. Viele wichtige Aufgaben des klinisch-ärztlichen Qualitätsmanagements (Behandlungsstandards, Indikationsboards, Morbidity-und-Mortality-Konferenzen, Registerführung usw.) sind weiterhin jedoch genauso fragmentiert wie die ärztlichen Führungsstrukturen. Jede ärztliche Klinik managt eigene Standards und Boards und die interdisziplinäre Abstimmung ist aufwendig. Es herrscht vielerorts eine frappante Diskrepanz zwischen dem zentralisierten und personell/professionell in der Regel gut ausgestatteten allgemeinen Qualitätsmanagement, sowie einer Vielfalt von dezentralen, häufig personell unterdotierten Lösungen im klinischen Qualitätsmanagement. Letzteres Fachärzten im Nebenamt aufzutragen, die primär am Patien- 
ten arbeiten sollten, oder dafür ungenügend qualifizierte Sekretariate oder Assistenzärzte einzusetzen, ist jedoch zunehmend untragbar.

Durch die Zentralisierung auch des klinischen Qualitätsmanagements und Besetzung mit qualifizierten Fachleuten wird dieses um einen bedeutenden Schritt professioneller und effizienter. Die Kommunikation der Ergebnisse wird objektiver und transparenter und die Wahrscheinlichkeit erhöht, dass Massnahmen zur Qualitätsverbesserung konsequent umgesetzt werden. Die Festlegung der Fachgebiets-spezifischen Behandlungs- und Qualitätsstandards verbleibt dabei ohne Abstriche in der Verantwortung der Leitungen der ärztlichen Service- oder Spezialisten-Einheiten. Diese Fachaufgabe kann ihnen keine übergeordnete Einheit abnehmen. Das zentrale klinische Qualitätsmanagement kann jedoch verlangen, dass Standards interdisziplinär so abgestimmt werden, dass der Behandlungspfad als Ganzes gemäss Best Practice und WZW-Kriterien (Wirksamkeit, Zweckmässigkeit, Wirtschaftlichkeit) gewährleistet ist. Während die fachliche Hoheit über die klinischen Behandlungs- und Qualitätsstandards also bei den Ärzten bleibt, wird das zentrale klinische Qualitätsmanagement die Bewirtschaftung massgeblich verbessern und die Verbindlichkeit erhöhen.

\section{Aufgaben der Spitalleitung}

Da unser Modell die Beschreibung von Aufgaben und Führungsprinzipien in den Vordergrund stellt, ist es nicht an eine bestimmte Aufbauorganisation gebunden. Die Umsetzung des Modells kann mit unterschiedlichen Organigrammen gelingen. So schreibt das Modell z.B. nicht vor, welche Bereiche, Professionen oder Funktionen in der Spitalleitung vertreten sein sollen. Dies ist für jedes Spital individuell zu entscheiden. Aus dem Modell leiten sich jedoch einige Empfehlungen für die obersten Führungsebenen des Spitals ab:

- Die operative Führung aller Medizinischen Service-Einheiten sollte hierarchisch geschehen und wird idealerweise unter eine gemeinsame Leitungsperson vereint, die aufgrund der hohen Bedeutung der Service-Einheiten Mitglied der Spitalleitung sein sollte.

- Die operative Führung der einzelnen Medizinischen Spezialisten- und Koordinations-Einheiten kann weniger eng durch vorgegebene Leistungs- und BudgetKennzahlen erfolgen, sollte aber nicht durch die Leitung der Service-Einheiten ausgeführt werden, um eine effektive Gewaltentrennung zu gewährleisten.

- Die operative Führung des Medizinischen Qualitätsund Performance-Managements erfolgt durch eine Leitungsperson, die bevorzugt direkt der Spitaldirektion zugeordnet ist. Sie sollte nicht der Leitung der
Service-Einheiten unterstellt sein, um eine effektive Gewaltentrennung zu gewährleisten. Allerdings muss die Leitungsperson einen offenen und transparenten Kontakt zu allen medizinischen Einheiten pflegen, um eine breite Akzeptanz zu gewährleisten.

- Die strategische Entwicklung der Medizinischen Service- und Spezialisten-Einheiten (Personal, Verfahren, Technologie, Infrastruktur) ist gleichzusetzen mit der Entwicklung des Leistungsportfolios des ganzen Spitals und sollte deshalb von der Spitaldirektion/-leitung geführt werden, wobei die enge Abstimmung mit und die Delegation von Entwicklungsprojekten an die Service- und SpezialistenEinheiten eine Selbstverständlichkeit sein sollte. Dazu stehen bereits heute Instrumente wie die «Zertifizierung von medizinischen Kompetenzzentren» der SQS zur Verfügung.

\section{Einführung des Modells}

Das hier beschriebene Systemmodell eignet sich nicht als fertiger Blueprint für die Neuorganisation von Spitälern. Vielmehr will das Modell Elemente und Prinzipien aufzeigen, die sich in der Klinik Hirslanden bewährt haben. Wir sind überzeugt, dass sich die beschriebenen Elemente verallgemeinern lassen und dass dieses Modell Spitälern, unabhängig von Rechtsform oder Trägerschaft, helfen kann, ihr Managementsystem leistungsfähiger zu machen. Entscheidend für die Einführung ist, dass Spitalleitung und Ärzteschaft sich von gewachsenen Strukturen und Rollenvorstellungen lösen, die ärztlichen Aufgaben klar definieren und diese arbeitsteilig den geeigneten Einheiten zuweisen. Jeder soll vor allem das machen, was er am besten kann.

Das Systemmodell macht keine Vorgaben für die Bezeichnung von Funktionseinheiten und Leitungsfunktionen. Bewährte Begriffe wie «Klinik», "Abteilung», "Chefarzt» oder "Leitender Arzt» können weiterhin verwendet werden, sollten jedoch auf ihre Bedeutungsinhalte überprüft und dem neuen Konzept angepasst werden. Organisation und Führung jeder Funktionseinheit soll ohne «Gärtchen-Denken» der spezifischen Aufgabe im Patientenprozess untergeordnet und angepasst werden. Kompetenzen für ärztliche Kernaufgaben sollten dabei an die jeweils tiefstmögliche Hierarchiestufe nahe am Patienten delegiert werden, während koordinative und administrative Aufgaben so weit wie möglich zu zentralisieren sind, um hochqualifizierten Fachärzten mehr Raum für ihre Kernaufgaben zu geben.
Literatur

1 Liedtke D, Wiesinger O, Westerhoff C, Stoffel U, Stocker R, Pahls S Ein innovatives Systemmodell für Akutspitäler. Schweiz Ärztezeitung. 2015;96(44):1625-8.

- Weitere Literatur beim Erstautor. 